\title{
"Birdlime" technique using TachoSil tissue sealing sheet soaked with fibrin glue for sutureless vessel transposition in microvascular decompression: operative technique and nuances
}

\author{
Naoki Otani, MD, PhD, ${ }^{1}$ Terushige Toyooka, MD, PhD, ${ }^{1}$ Kazuya Fujii, MD, PhD, ${ }^{1}$ \\ Kosuke Kumagai, MD, ${ }^{1}$ Satoru Takeuchi, MD, PhD, ${ }^{1}$ Arata Tomiyama, MD, PhD, ${ }^{1}$ \\ Yasuaki Nakao, MD, PhD, ${ }^{2}$ Takuji Yamamoto, MD, PhD, ${ }^{2}$ Kojiro Wada, MD, PhD, ${ }^{1}$ and \\ Kentaro Mori, MD, PhD'
}

\begin{abstract}
'Department of Neurosurgery, National Defense Medical College, Tokorozawa, Saitama; and 2Department of Neurosurgery, Juntendo University Shizuoka Hospital, Izunokuni, Shizuoka, Japan
\end{abstract}

\begin{abstract}
OBJECTIVE Microvascular decompression (MVD) is effective for the treatment of trigeminal neuralgia (TN), hemifacial spasm (HFS), and glossopharyngeal neuralgia. The transposition technique is the standard procedure to avoid adhesions and granuloma around the decompression site but is more complex and difficult to perform than the interposition technique. The authors describe a simple and safe MVD transposition procedure they call the "birdlime" technique, which uses a tissue glue-coated collagen sponge soaked with fibrin glue, and the results of this technique.
\end{abstract}

METHODS The authors retrospectively reviewed the medical charts and radiographic findings of 27 consecutive patients with TN (8 patients) and HFS (19 patients) who, between January 2012 and December 2015, had undergone an MVD transposition procedure utilizing a tissue glue-coated collagen sponge (TachoSil tissue sealing sheet) soaked with fibrin glue (Tisseel 2-component fibrin sealant, vapor heated). Offending arteries among the patients with TN were the superior cerebellar artery (SCA) in 5 patients, the SCA and anterior inferior cerebellar artery (AICA) in 2, and the AICA in 1. Those among the patients with HFS were the vertebral artery (VA) in 3 patients, the VA and AICA in 4, the VA and posterior inferior cerebellar artery (PICA) in 3, the PICA in 4, the AICA in 1, the AICA-PICA in 3, and the PICA and AICA in 1. Operations were performed according to the Jannetta procedure. The offending artery was transposed and fixed to the dura mater of the petrous bone using TachoSil pieces soaked with fibrin glue. Postoperative constructive interference in steady-state MRI was performed to evaluate the change in the position of the offending artery.

RESULTS Transposition of the offending artery was easily and safely performed in all patients. All patients had total remission of symptoms directly after the procedure. No severe complications occurred. The postoperative course was uneventful. No recurrences, adhesions, or dysfunction of the cranial nerves was observed in any of the patients. Postoperative MRI showed that the offending vessels were displaced and fixed in the appropriate position.

CONCLUSIONS The described transposition technique provides an easy and adjustable way to perform MVD safely and effectively. In addition, this transposition and fixation technique is simple and avoids the risk of needle injury close to the cranial nerves and vessels. This simple sutureless technique is recommended for MVD to reduce the risk of intraoperative neurovascular injury.

https://thejns.org/doi/abs/10.3171/2017.1.JNS161243

KEY WORDS microvascular decompression; vessel transposition; TachoSil; fibrin glue; functional neurosurgery; surgical technique

$\mathrm{M}$ ICROVASCULAR decompression (MVD) is an effective surgical procedure for the treatment of hemifacial spasm (HFS), trigeminal neuralgia (TN), and glossopharyngeal neuralgia. ${ }^{10}$ Recent advances in microsurgical techniques have resulted in further improve- ments in the outcome of MVD. Therefore, current surgical management for MVD focuses on minimizing recurrence rates and reducing operative morbidity and mortality.

Two different techniques are used for displacing the offending vessels: the interposition method and the trans-

ABBREVIATIONS AICA = anterior inferior cerebellar artery; HFS = hemifacial spasm; ICG = indocyanine green; MVD = microvascular decompression; PICA = posterior inferior cerebellar artery; REZ = root exit zone; $\mathrm{SCA}$ = superior cerebellar artery; TN = trigeminal neuralgia; VA = vertebral artery.

SUBMITTED May 13, 2016. ACCEPTED January 19, 2017.

INCLUDE WHEN CITING Published online August 11, 2017; DOI: 10.3171/2017.1.JNS161243. 

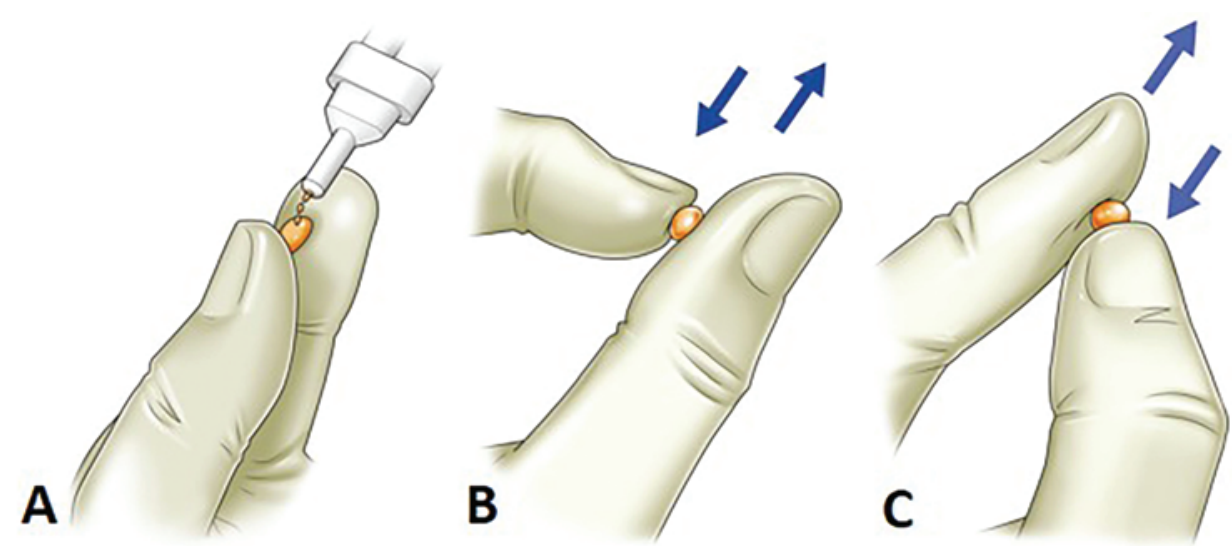

FIG. 1. Illustration showing how to prepare the adhesive material, similar to birdlime, which was trimmed and soaked with fibrin glue between thumb and forefinger. Copyright Naoki Otani. Published with permission.

position method. A favorable surgical outcome following MVD depends on permanent and complete transposition of the offending vessels. ${ }^{23}$ However, current transposition procedures are more complicated, more time consuming, and more hazardous than interposition techniques because suturing with a needle is required in the vicinity of the brainstem. ${ }^{3,5,15,18,20,25,26}$

The TachoSil tissue sealing sheet (CSL Behring K.K.) is a ready-to-use surgical patch consisting of a white collagen sponge coated on one side with fibrinogen and thrombin, which has been used in various neurosurgical procedures to achieve hemostasis ${ }^{7}$ and tissue sealing to prevent CSF leakage. ${ }^{8,11}$ Experience with this material in MVD was first described in 2011 as the "double-stick tape" technique.? We modified this technique to improve ease of handling and performance of TachoSil, which we trimmed and soaked with fibrin glue between thumb and forefinger to create an adhesive material similar to birdlime (Fig. 1), an adhesive and sticky material smeared on twigs to ensnare small birds. We here describe our simple, quick transposition method using TachoSil in MVD and evaluate the outcomes with special emphasis on the clinical and surgical outcomes in a series of 27 consecutive patients. We discuss the surgical advantages and pitfalls of this newly modified, sutureless transposition method for MVD.

\section{Methods \\ Patient Characteristics}

This retrospective analysis included 27 consecutive patients, 19 with HFS and 8 with TN, whose ages ranged from 41 to 73 years (mean 62 years) and who had undergone surgical treatment at the National Defense Medical College Hospital between January 2012 and December 2015. Microvascular decompression was performed via a conventional lateral suboccipital retroauricular approach. Medical charts, radiological findings, surgical techniques, complications, and final surgical results were retrospectively reviewed. At discharge, patient outcome was assessed as good recovery, moderate disability, severe disability, vegetative state, and death, according to the Glasgow Outcome Scale. All patients underwent MRI and MR angiography before and after the operation. All patients underwent de- tailed pre- and postoperative evaluations of hearing function by an otolaryngologist.

\section{Surgical Material}

A standard sponge TachoSil tissue sealing sheet was used in all procedures. This product has an active yellow side coated with human thrombin $\left(2.0 \mathrm{IU} / \mathrm{cm}^{2}\right)$ and human fibrinogen $\left(5.5 \mathrm{mg} / \mathrm{cm}^{2}\right)$ and a supportive white side coated with collagen. The sheet was trimmed with surgical scissors and soaked with fibrin glue (Tisseel 2-component fibrin sealant, vapor heated, Baxter Healthcare Corp.) between thumb and forefinger to create an adhesive material similar to birdlime (Fig. 1).

\section{Operative Procedure}

All patients underwent a standard retrosigmoid approach under auditory brainstem response monitoring. All patients were placed in the lateral decubitus position, and the head was fixed on the operating table and turned $30^{\circ}$ to the contralateral side, with the space of 1 finger breadth between the chin and neck. A retromastoid curvilinear skin incision was made. The mastoid eminence, digastric groove, and inion were identified to define the transverse sigmoid junction.

To treat TN, a craniotomy is performed to expose the border of the transverse and sigmoid sinuses. The spatula is placed over the marginal aspect of the cerebellar surface. With slight elevation of the cerebellum without injury to the petrosal vein, the trigeminal nerve can be seen. Offending vessels are dissected and mobilized away from the trigeminal nerve. To decompress the offending vessels, the adhesive TachoSil material is placed on the tentorial or dural surface of the petrous bone, depending on the required direction of the transposition, and the offending vessels are displaced with microfine forceps for 3 minutes. The surgical procedure is shown in Fig. 2. After all adjustments are completed, the adhesive site is additionally secured with fibrin glue. Finally, indocyanine green (ICG) angiography is performed to confirm patency of the offending vessels and perforators. The dura mater is closed in a watertight fashion. The wound is closed layer by layer.

To treat HFS, the supracondylar area is removed to min- 

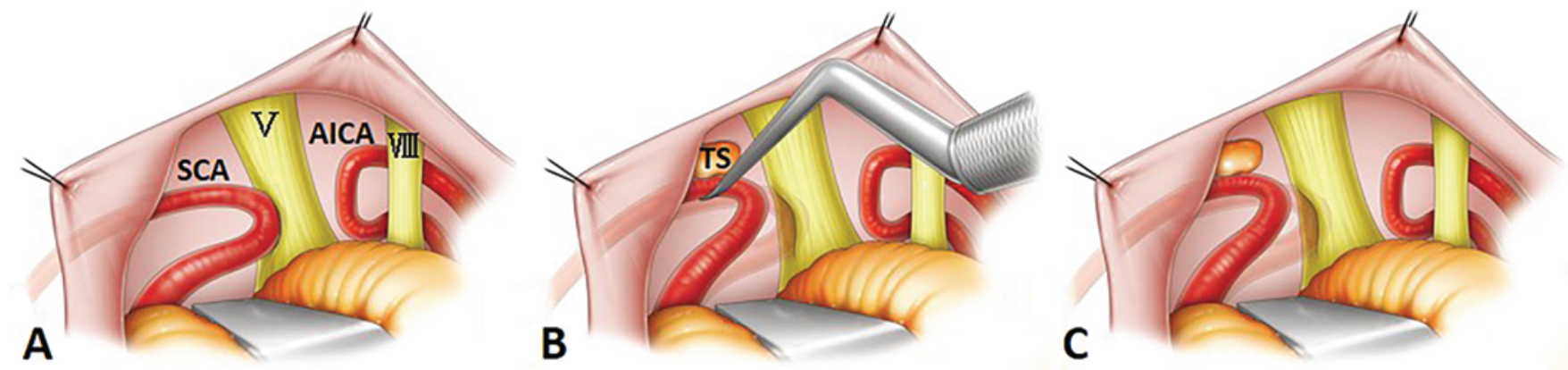

FIG. 2. Illustrations of transposition using the TachoSil material. A: Offending vessels are dissected and mobilized away from the trigeminal nerve. B: The adhesive TachoSil (TS) material is placed on the tentorial or dural surface of the petrous bone, depending on the required direction of the transposition, and the offending vessels are displaced with microfine forceps for 3 minutes. C: After all adjustments are completed, vessel transposition is confirmed. $\mathrm{V}=$ trigeminal nerve; $\mathrm{VIII}=$ acoustic nerve. Copyright Naoki Otani. Published with permission.

imize retraction and to approach the root exit zone (REZ) of the facial nerve directly from the caudal direction. The dural incision is curvilinear. The arachnoid membrane of the cisterna magna is incised, and the CSF is drained sufficiently to provide adequate cerebellar relaxation. The spatula is placed over the inferior aspect of the cerebellar surface. With slight elevation of the inferior medial surface of the cerebellum, the entire courses of the ninth and 10th cranial nerves can be seen. Advanced arachnoid dissection of the cerebellomedullary fissure along the proximal portion of the lower cranial nerves toward their origins exposes the choroid plexus of the lateral recess of the fourth ventricle. With slight elevation of the flocculus and the choroid plexus away from the ninth cranial nerve, the REZ of the facial nerve can easily be observed without tension on the eighth cranial nerve. Offending vessels are carefully visualized, dissected, and mobilized away from the REZ of the facial nerve. To decompress the offending vessels, the adhesive TachoSil material is placed on the posterior dural surface of the petrous bone, and the offending vessels are displaced with microfine forceps for 3 minutes. The procedure is completed as described for TN.

\section{Results}

The clinical characteristics of the patients are summarized in Table 1. This transposition method was used to achieve MVD in 19 patients with HFS and 8 patients with TN. A torturous vertebral artery (VA) was transposed in 10 HFS patients, and the anterior inferior cerebellar artery (AICA) or posterior inferior cerebellar artery (PICA) was transposed in 9 patients. Multiple offending vessels were transposed in 2 TN patients, and a single offending AICA or superior cerebellar artery (SCA) was transposed in 6 . Fixation of the offending vessels with TachoSil and fibrin glue was performed in all patients. No permanent complications related to the surgical procedure occurred, nor did any infections or allergic reactions. Hemifacial spasm disappeared within 3 months in 18 of the 19 patients. During a follow-up of 17 months, 1 patient suffered a recurrence of HFS due to compression of another offending artery at the distal site of the facial nerve. Trigeminal neuralgia disappeared within 3 months in 7 of the 8 patients. One patient had slight numbness after surgery caused by the petrosal vein. There were no surgery-related deaths or lifethreatening complications throughout the study period.

There were no patients who had recurrence of $\mathrm{TN}$ or HFS due to the birdlime technique during a mean follow-up of 28.8 months (9-56 months). Preoperative MRI showed the offending vessels compressing the REZ and/or trigeminal nerve. In all patients, follow-up MRI confirmed that the offending vessels were displaced with the adhesive TachoSil material.

\section{Illustrative Cases}

\section{Case 24}

\section{Examination}

A 66-year-old woman had suffered daily TN for 8 years. Medication gradually became ineffective. Neurological examination on admission showed no abnormalities. Preoperative MRI revealed that the right SCA and AICA compressed the trigeminal nerve at the cisternal portion.

\section{Operation}

Microvascular decompression was performed via the lateral suboccipital approach. The entire course of the cisternal portion of the trigeminal nerve was completely exposed, and the SCA and AICA were found to adhere to and compress the trigeminal nerve, resulting in an indentation on the nerve (Fig. 3A and B). TachoSil pieces soaked with fibrin glue were placed on the dural surface of the petrous bone. Until the glue hardened, the SCA was pressed onto the TachoSil on the dura of the petrous bone for 3 minutes. After this procedure, SCA transposition was confirmed (Fig. 3C). In addition, a TachoSil piece soaked with fibrin glue was placed on the tentorial surface. The AICA was pressed onto the TachoSil on the tentorial surface for 3 minutes. After this procedure, AICA transposition was confirmed (Fig. 3D). Electrophysiological monitoring showed no changes during the operation.

\section{Postoperative Course}

The postoperative course was uneventful, and the patient's symptoms completely resolved immediately after 
TABLE 1. Clinical characteristics of 27 patients who underwent vessel transposition using TachoSil tissue sealing sheet soaked with fibrin glue for MVD

\begin{tabular}{|c|c|c|c|c|c|c|}
\hline Case No. & Age (yrs)/Sex & Side & Symptom & Offending Artery & Surgical Complication & Surgical Outcome \\
\hline 1 & $73 / F$ & $\mathrm{Lt}$ & HFS & VA \& AICA & None & $C R$ \\
\hline 2 & $49 / F$ & $\mathrm{Lt}$ & HFS & AICA & None & $C R$ \\
\hline 3 & $43 / \mathrm{F}$ & $\mathrm{Rt}$ & HFS & AICA-PICA & None & CR \\
\hline 4 & $58 / \mathrm{F}$ & $\mathrm{Rt}$ & HFS & PICA \& AICA & None & $\mathrm{CR}$ \\
\hline 5 & $60 / \mathrm{F}$ & $\mathrm{Rt}$ & HFS & VA & None & $\mathrm{CR}$ \\
\hline 6 & $70 / \mathrm{M}$ & Lt & HFS & VA & None & $\mathrm{CR}$ \\
\hline 7 & $61 / \mathrm{F}$ & $\mathrm{Lt}$ & HFS & Bilat VA & None & $\mathrm{CR}$ \\
\hline 8 & $41 / \mathrm{F}$ & $\mathrm{Lt}$ & HFS & AICA-PICA & None & $\mathrm{CR}$ \\
\hline 9 & $53 / F$ & $\mathrm{Rt}$ & HFS & PICA & None & $50 \%$ improvement \\
\hline 10 & $66 / F$ & Lt & HFS & PICA & None & CR \\
\hline 11 & $46 / F$ & $\mathrm{Lt}$ & HFS & VA \& PICA & None & $C R$ \\
\hline 12 & $43 / \mathrm{M}$ & $\mathrm{Lt}$ & HFS & VA \& AICA & None & $C R$ \\
\hline 13 & $59 / \mathrm{M}$ & $\mathrm{Lt}$ & HFS & VA \& AICA & None & $\mathrm{CR}$ \\
\hline 14 & $53 / \mathrm{M}$ & $\mathrm{Rt}$ & HFS & AICA-PICA & None & $\mathrm{CR}$ \\
\hline 15 & $62 / \mathrm{M}$ & Lt & HFS & VA \& AICA & None & $\mathrm{CR}$ \\
\hline 16 & $57 / \mathrm{M}$ & $\mathrm{Lt}$ & HFS & VA \& PICA, AICA & None & $\mathrm{CR}$ \\
\hline 17 & $60 / \mathrm{M}$ & $\mathrm{Lt}$ & HFS \& GPN & VA \& PICA, VA & Transient HD & $\mathrm{CR}$ \\
\hline 18 & $68 / \mathrm{F}$ & $\mathrm{Lt}$ & HFS & PICA & None & $\mathrm{CR}$ \\
\hline 19 & $59 / \mathrm{M}$ & $\mathrm{Rt}$ & HFS & PICA & None & $C R$ \\
\hline 20 & $58 / \mathrm{M}$ & $\mathrm{Rt}$ & TN (V3) & SCA & None & $\mathrm{CR}$ \\
\hline 21 & $70 / \mathrm{F}$ & $\mathrm{Rt}$ & TN (V2) & SCA \& PV & None & $80 \%$ improvement \\
\hline 22 & $47 / F$ & Lt & TN (V3-2) & SCA & None & $\mathrm{CR}$ \\
\hline 23 & $65 / F$ & $\mathrm{Lt}$ & TN (V3) & SCA & None & $\mathrm{CR}$ \\
\hline 24 & $66 / F$ & $\mathrm{Rt}$ & TN (V3) & SCA \& AICA & None & $\mathrm{CR}$ \\
\hline 25 & $68 / \mathrm{M}$ & $\mathrm{Rt}$ & TN (V3-2) & AICA & None & $\mathrm{CR}$ \\
\hline 26 & $72 / \mathrm{M}$ & Lt & TN (V3-2) & SCA \& AICA & None & $C R$ \\
\hline 27 & $60 / \mathrm{M}$ & $\mathrm{Lt}$ & TN (V3-2) & SCA & None & $\mathrm{CR}$ \\
\hline
\end{tabular}

$\mathrm{CR}=$ complete remission; $\mathrm{GPN}=$ glossopharyngeal neuralgia; $\mathrm{HD}=$ hearing disturbance; $\mathrm{PV}$ = petrosal vein .

surgery. The patient was discharged with no neurological deficits.

\section{Case 15}

\section{Examination}

A 62-year-old man presented with a 3-year history of left-sided facial spasm including the orbicularis oculi and orbicularis oris muscles, triggered by cold or effort stimulation. Preoperative MRI revealed a tortuous left VA indenting into the REZ (Fig. 4A and D).

\section{Operation}

The patient underwent MVD via the transcondylar fossa approach to gain wide exposure of the REZ of the facial nerve from the caudal side and to minimize cerebellum retraction. The offending VA was dissected away from the pontomedullary junction toward the cranial base, and the AICA and VA were found to adhere and compress the facial nerve, resulting in an indentation on the nerve (Fig. 5A). A TachoSil piece was placed on the petrosal surface of the jugular tubercle, and the VA was pressed onto the TachoSil on the petrous bone (Fig. 5B). Thereafter, VA transposition was confirmed (Fig. 5C). TachoSil pieces soaked with fibrin glue were placed onto the dural surface of the petrous bone. Until the glue hardened, the VA was pressed onto the TachoSil on the dura of the petrous bone for 3 minutes. After this procedure, complete VA transposition was confirmed (Fig. 5D). In addition, a TachoSil piece soaked with fibrin glue was placed on the VA, and the offending PICA was pressed onto the TachoSil for adhesion with the VA. After this procedure, PICA transposition was confirmed (Fig. 5E). Electrophysiological monitoring demonstrated no changes during the operation. To confirm the patency of the offending vessels and perforators, ICG angiography was performed (Fig. 5F).

\section{Postoperative Course}

The patient's postoperative course was uneventful. His symptoms completely resolved immediately after surgery. Postoperative MRI showed the VA was completely transposed away from the REZ (Fig. 4B). TachoSil adhered to the dural surface of the petrous bone (Fig. 4E). The patient was discharged with no neurological deficits, and he remained asymptomatic at his 2-year follow-up visit. In ad- 

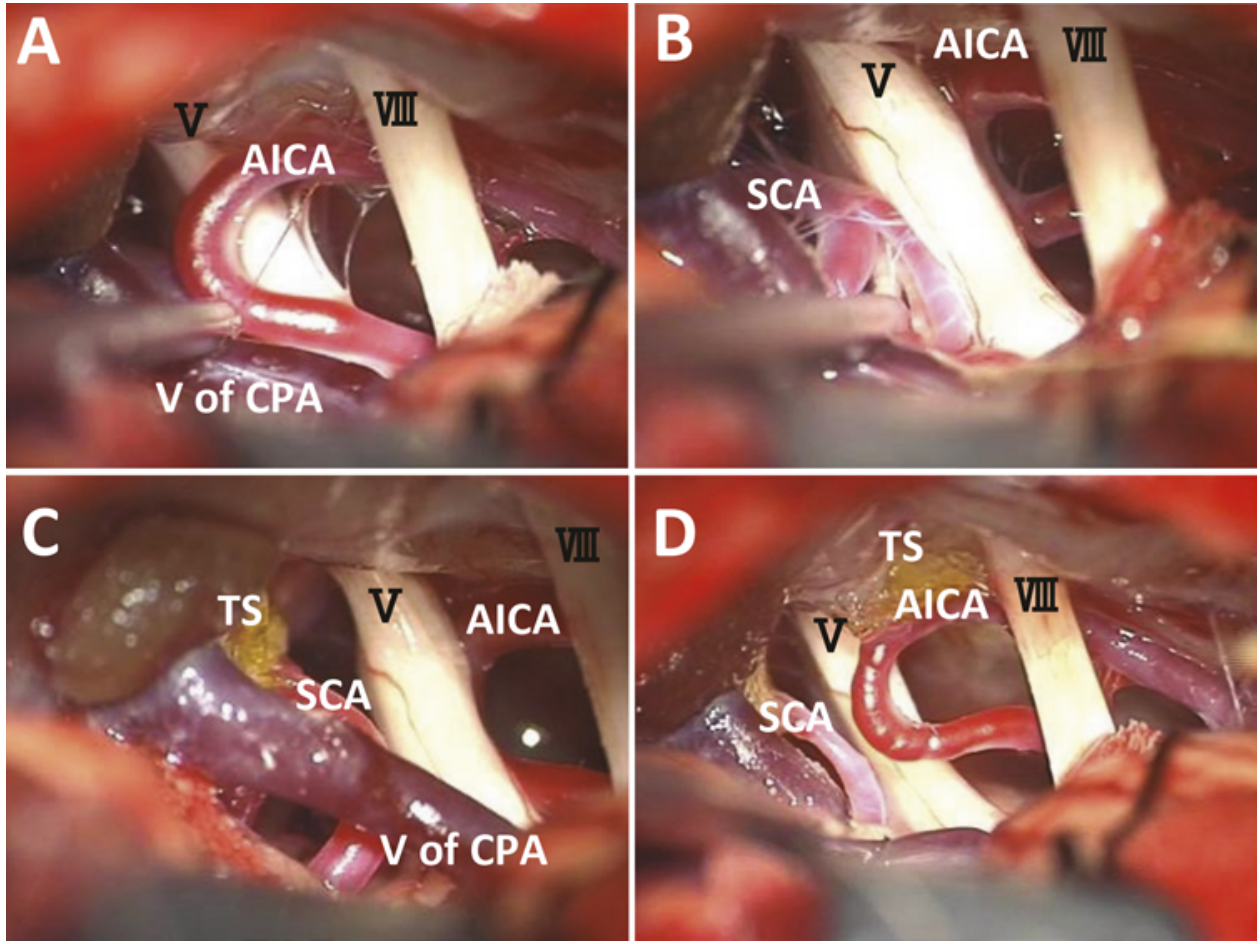

FIG. 3. Case 24. Microvascular decompression was performed via the lateral suboccipital approach. The entire course of the cisternal portion of the trigeminal nerve was completely exposed, and the SCA and AICA were found to adhere to and compress the trigeminal nerve, resulting in an indentation on the nerve (A and B). TachoSil (TS) pieces soaked with fibrin glue were placed on the dural surface of the petrous bone. Until the glue hardened, the SCA was pressed onto the TachoSil on the dura of the petrous bone for 3 minutes. After this procedure, SCA transposition was confirmed (C). In addition, a TachoSil piece soaked with fibrin glue was placed on the tentorial surface. The AICA was pressed onto the TachoSil on the tentorial surface for 3 minutes. After this procedure, AICA transposition was confirmed (D). $\mathrm{V}=$ trigeminal nerve; $\mathrm{V}$ of $\mathrm{CPA}=$ vein of the cerebellopontine angle.
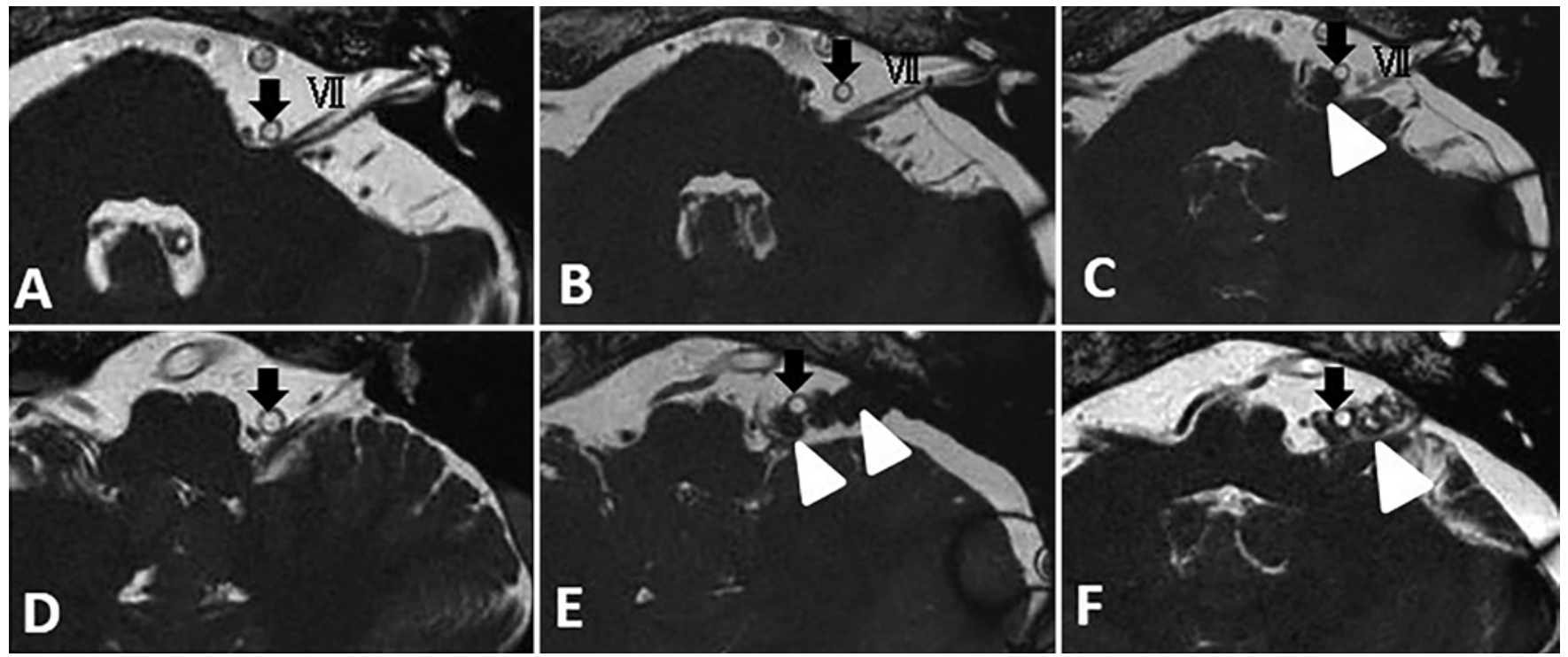

FIG. 4. Case 15. A and D: Preoperative MR images revealing a tortuous left VA (black arrows) indenting into the REZ. B and E: Postoperative MR images showing the VA (black arrows) was completely transposed away from the REZ. TachoSil (white arrowheads) adhered to the dural surface of the petrous bone. C and F: Follow-up MR images obtained 2 years after surgery, indicating that the VA (black arrows) as an offending vessel was displaced with adhesive TachoSil material (white arrowheads), which showed no infectious and/or inflammatory reaction. VII = facial nerve. 


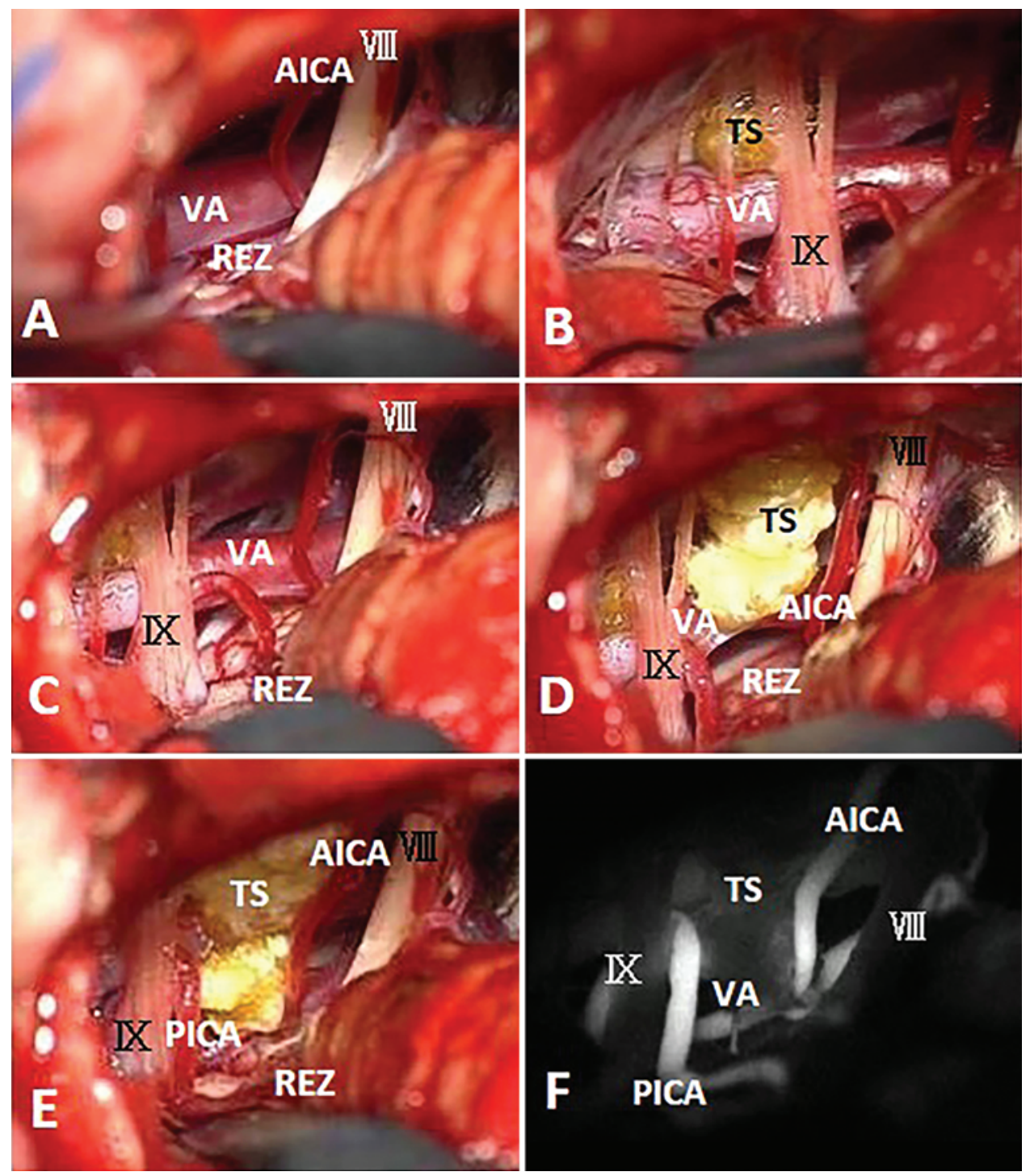

FIG. 5. Case 15. Intraoperative photograph showing the offending VA dissected away from the pontomedullary junction toward the cranial base, and the AICA and VA adhering to and compressing the facial nerve, resulting in an indentation on the nerve (A). A TachoSil (TS) piece was placed on the petrosal surface of the jugular tubercle, and the VA was pressed onto the TachoSil on the petrous bone (B). Thereafter, VA transposition was confirmed (C). TachoSil pieces soaked with fibrin glue were placed onto the dural surface of the petrous bone. Until the glue hardened, the VA was pressed onto the TachoSil on the dura of the petrous bone for 3 minutes. After this procedure, complete VA transposition was confirmed (D). In addition, a TachoSil piece soaked with fibrin glue was placed on the VA, and the offending PICA was pressed to form adhesion with the VA. After this procedure, PICA transposition was confirmed $(E)$. Indocyanine green angiography was performed to confirm the patency of the offending vessels and perforators (F). VIII = acoustic nerve; IX = glossopharyngeal nerve.

dition, follow-up MRI 2 years after surgery indicated that the VA as an offending vessel was displaced with adhesive TachoSil material (Fig. 4C), which showed no infectious and/or inflammatory reaction (Fig. 4F).

\section{Discussion}

Vascular compression in patients with HFS was first described by Campbell and Keedy in 1947. ${ }^{4}$ Vascular decompression for the treatment of HFS was proposed by Gardner and Sava. ${ }^{6}$ Jannetta et al. suggested that the etiology of HFS was vascular compression at the REZ of the facial nerve. ${ }^{10}$ During the last decades, advances in mi- crosurgical techniques have improved MVD's outcomes and reduced its operative morbidity and mortality. Previously, the incidence of permanent cranial nerve injury was $10 \%$ in patients treated with MVD. ${ }^{19}$ And, MVD in 648 patients with HFS resulted in postoperative permanent facial palsy in 22 patients (3.4\%) and hearing loss in 21 patients $(3.3 \%){ }^{2}$ These complications from conventional MVD may be related to the mass effect introduced by the interposed prosthetic material positioned during surgery.

The 2 techniques for shifting the offending vessels are the interposition method and the transposition method. Favorable surgical outcomes of MVD depend on permanent 
and complete transposition of the offending vessels. ${ }^{23}$ Sindou et al. suggested that the significant, better long-term cure rate when an implant is not in contact with the nerve root favors the pure decompressive effect of the MVD procedure rather than a conduction block mechanism. In terms of the outcome, foreign material touching the nerve may produce a harmful neocompressive effect and hamper the long-term efficacy of the procedure..$^{21,22}$ The first transposition method for treating HFS used Teflon tape. ${ }^{5}$ Various materials have since been employed in transposition procedures, including strips of dura, ${ }^{3,12}$ fascia, ${ }^{17}$ sutures, ${ }^{3,18}$ tapes, ${ }^{16}$ fibrin tissue-adhesive collagen fleece product, ${ }^{14}$ silicone (polydimethylsiloxane) tube, ${ }^{25}$ fenestrated aneurysm clip, ${ }^{1}$ or expanded polytetrafluoroethylene. ${ }^{18,20}$ However, these transposition procedures are more complicated, more time consuming, and more hazardous than interposition techniques because suturing with a needle is required in the vicinity of the brainstem. $3,5,15,18,20,25,26$

Transposition in the present series utilized the TachoSil tissue sealing sheet, classified as a topical hemostat, sealant, and adhesive, ${ }^{24}$ which is a fixed combination of collagen matrix coated with 2 coagulation factors, fibrinogen and thrombin, and indicated as a supportive treatment for the improvement of hemostasis to promote tissue sealing. Kivelev et al. ${ }^{13}$ retrospectively analyzed 100 consecutive patients who had undergone microneurosurgical procedures utilizing TachoSil and reported that no patient showed any signs of allergic reaction or infection. Microvascular decompression using TachoComb was first described by Ichikawa et al. ${ }^{9}$ in 2011. TachoSil has several advantages for MVD given its elasticity, ease in manipulating it into various shapes and configurations, and easy application. In addition, this material only requires adhesive attachment to the dura of the petrous or clival bone and to the tentorium. Therefore, our technique can avoid the risk of injury from both suturing and excessive cerebellar retraction and is less complex, less hazardous, and quicker than previously reported techniques. The application of TachoSil as we describe it here requires careful attention to prevent vascular kinking or stenosis of the transposed vessels and preservation of the brainstem perforators, which can be assessed by direct observation as well as by intraoperative Doppler flow and ICG video angiography. If the transposed vessel is seen to be in a suboptimal position, for example, causing vessel kinking, the vessels can be easily separated from the implant material and repositioned without tissue damage, even numerous times. Therefore, we can reposition the offending vessels until we find their optimal placement without causing vessel kinking and/or stenosis.

In particular, the characteristics of this material have been beneficial for transposition of the VA. In the present series, 10 patients underwent safe transposition of the VA as an offending vessel. This material can be easily applied and even stacked because of its elasticity and the ease in manipulating it into various shapes and configurations (Fig. 5B-E) in deep and narrow operative sites, such as the jugular tubercle, through the inferior cranial nerves. Therefore, meticulous VA transposition can be achieved with the natural shape and direction of the vessel with as little tensile force at the localized vessel wall as possible.

\section{Conclusions}

A series of 27 consecutive patients with HFS were treated with MVD using TachoSil soaked with fibrin glue, which has several advantages in terms of its elasticity, ease in manipulating it into various shapes and configurations, and easy application. Our modified, simple, quick method can be used to perform vessel transposition easily and precisely, minimizing postoperative complications. We recommend this sutureless technique for vessel transposition in MVD to reduce the risk of intraoperative neurovascular injury.

\section{References}

1. Attabib N, Kaufmann AM: Use of fenestrated aneurysm clips in microvascular decompression surgery. Technical note and case series. J Neurosurg 106:929-931, 2007

2. Barker FG II, Jannetta PJ, Bissonette DJ, Shields PT, Larkins MV, Jho HD: Microvascular decompression for hemifacial spasm. J Neurosurg 82:201-210, 1995

3. Bejjani GK, Sekhar LN: Repositioning of the vertebral artery as treatment for neurovascular compression syndromes. Technical note. J Neurosurg 86:728-732, 1997

4. Campbell E, Keedy C: Hemifacial spasm; a note on the etiology in two cases. J Neurosurg 4:342-347, 1947

5. Fukushima T: [Results of posterior fossa microvascular decompression in the treatment of hemifacial spasm.] Facial Nerve Res Jpn 4:9-14, 1984 (Jpn)

6. Gardner WJ: Concerning the mechanism of trigeminal neuralgia and hemifacial spasm. J Neurosurg 19:947-958, 1962

7. Gazzeri R, Galarza M, Fiore C, Callovini G, Alfieri A: Use of tissue-glue-coated collagen sponge (TachoSil) to repair minor cerebral dural venous sinus lacerations: technical note. Neurosurgery 11 (Suppl 2):32-36, 2015

8. Hutter G, von Felten S, Sailer MH, Schulz M, Mariani L: Risk factors for postoperative CSF leakage after elective craniotomy and the efficacy of fleece-bound tissue sealing against dural suturing alone: a randomized controlled trial. J Neurosurg 121:735-744, 2014

9. Ichikawa T, Agari T, Kurozumi K, Maruo T, Satoh T, Date I: "Double-stick tape" technique for transposition of an offending vessel in microvascular decompression: technical case report. Neurosurgery 68 (2 Suppl Operative):377-382, 2011

10. Jannetta PJ, Abbasy M, Maroon JC, Ramos FM, Albin MS: Etiology and definitive microsurgical treatment of hemifacial spasm. Operative techniques and results in 47 patients. J Neurosurg 47:321-328, 1977

11. Kamenova M, Leu S, Mariani L, Schaeren S, Soleman J: Management of incidental dural tear during lumbar spine surgery. To suture or not to suture? World Neurosurg 87:455-462, 2016

12. Khoo HM, Yoshimine T, Taki T: A "sling swing transposition" technique with pedicled dural flap for microvascular decompression in hemifacial spasm. Neurosurgery 71 (1 Suppl Operative):25-30, 2012

13. Kivelev J, Göhre F, Niemelä M, Hernesniemi J: Experiences with TachoSil ${ }^{\circledR}$ in microneurosurgery. Acta Neurochir (Wien) 157:1353-1357, 2015

14. Kurokawa Y, Maeda Y, Toyooka T, Inaba K: Microvascular decompression for hemifacial spasm caused by the vertebral artery: a simple and effective transposition method using surgical glue. Surg Neurol 61:398-403, 2004

15. Kyoshima K, Watanabe A, Toba Y, Nitta J, Muraoka S, Kobayashi S: Anchoring method for hemifacial spasm associated with vertebral artery: technical note. Neurosurgery 45:1487-1491, 1999 
16. Lin CF, Chen HH, Hernesniemi J, Lee CC, Liao CH, Chen $\mathrm{SC}$, et al: An easy adjustable method of ectatic vertebrobasilar artery transposition for microvascular decompression. Clin Neurol Neurosurg 114:951-956, 2012

17. Mitsos AP, Georgakoulias N, Lafazanos SA, Konstantinou EA: The "hanging technique" of vascular transposition in microvascular decompression for trigeminal neuralgia: technical report of four cases. Neurosurg Rev 31:327-330, 2008

18. Ogawa A, Suzuki M, Shirane R, Yoshimoto T: Repositioning of the tortuous vertebrobasilar artery for trigeminal neuralgia: a technical note. Surg Neurol 38:232-235, 1992

19. Piatt JH Jr, Wilkins RH: Treatment of tic douloureux and hemifacial spasm by posterior fossa exploration: therapeutic implications of various neurovascular relationships. Neurosurgery 14:462-471, 1984

20. Shigeno T, Kumai J, Endo M, Oya S, Hotta S: Snare technique of vascular transposition for microvascular decompression-technical note. Neurol Med Chir (Tokyo) 42:184-190, 2002

21. Sindou M, Amrani F, Mertens P: Does microsurgical vascular decompression for trigeminal neuralgia work through a neo-compressive mechanism? Anatomical-surgical evidence for a decompressive effect. Acta Neurochir Suppl (Wien) 52:127-129, 1991

22. Sindou M, Leston J, Decullier E, Chapuis F: Microvascular decompression for primary trigeminal neuralgia: long-term effectiveness and prognostic factors in a series of 362 consecutive patients with clear-cut neurovascular conflicts who underwent pure decompression. J Neurosurg 107:1144-1153, 2007

23. Sindou M, Leston JM, Decullier E, Chapuis F: Microvascular decompression for trigeminal neuralgia: the importance of a noncompressive technique-Kaplan-Meier analysis in a con- secutive series of 330 patients. Neurosurgery 63 (4 Suppl 2):341-351, 2008

24. Spotnitz WD: Fibrin sealant: past, present, and future: a brief review. World J Surg 34:632-634, 2010

25. Stone JL, Lichtor T, Crowell RM: Microvascular sling decompression for trigeminal neuralgia secondary to ectatic vertebrobasilar compression. Case report. J Neurosurg 79:943-945, 1993

26. Suzuki S, Tsuchita H, Kurokawa Y, Kitami K, Sohma T, Takeda T: New method of MVD using a vascular tape for neurovascular compression involving the vertebrobasilar artery-report of two cases. Neurol Med Chir (Tokyo) 30:1020-1023, 1990

\section{Disclosures}

The authors report no conflict of interest concerning the materials or methods used in this study or the findings specified in this paper.

\section{Author Contributions}

Conception and design: Otani. Acquisition of data: Takeuchi. Analysis and interpretation of data: Fujii. Drafting the article: Tomiyama, Kumagai. Statistical analysis: Toyooka, Nakao, Yamamoto. Study supervision: Wada, Mori.

\section{Correspondence}

Naoki Otani, Department of Neurosurgery, National Defense Medical College, 3-2 Namiki, Tokorozawa, Saitama 359-8513, Japan.email: naotani@ndmc.ac.jp. 